\title{
Constraints on the generalized Chaplygin gas model including gamma-ray bursts via a Markov Chain Monte Carlo approach
}

\author{
N. Liang ${ }^{1}$, L. Xu ${ }^{2,3}$, and Z.-H. Zhu ${ }^{1}$ \\ 1 Department of Astronomy, Beijing Normal University, Beijing 100875, PR China \\ e-mail: [liangn; zhuzh]@bnu.edu.cn \\ 2 School of Physics and Optoelectronic Technology, Dalian University of Technology, Dalian, Liaoning 116024, PR China \\ e-mail: lxxu@dlut.edu.cn \\ 3 College of Advanced Science \& Technology, Dalian University of Technology, Dalian, 116024, PR China \\ Received 13 October 2010 / Accepted 29 November 2010
}

ABSTRACT

\begin{abstract}
Aims. We investigate observational constraints on the generalized Chaplygin gas (GCG) model including the gamma-ray bursts (GRBs) at high redshift obtained directly from the Union2 type Ia supernovae (SNe Ia) set.

Methods. By using the Markov Chain Monte Carlo method, we constrain the GCG model with the cosmology-independent GRBs, as well as the Union2 set, the cosmic microwave background (CMB) observation from the Wilkinson Microwave Anisotropy Probe (WMAP7) result, and the baryonic acoustic oscillation (BAO) observation from the spectroscopic Sloan Digital Sky Survey (SDSS) data release 7 (DR7) galaxy sample.

Results. The best-fit values of the GCG model parameters are $A_{\mathrm{S}}=0.7475_{-0.0539}^{+0.0556}(1 \sigma)_{-0.0816}^{+0.0794}(2 \sigma), \alpha=-0.0256_{-0.1326}^{+0.1760}(1 \sigma)_{-0.1907}^{+0.2730}(2 \sigma)$, and the effective matter density $\Omega_{\mathrm{m}}=0.2629_{-0.0153}^{+0.0155}(1 \sigma)_{-0.0223}^{+0.0236}(2 \sigma)$, which are more stringent than previous results for constraining GCG model parameters.
\end{abstract}

Key words. cosmological parameters - gamma rays: galaxies

\section{Introduction}

The original Chaplygin gas (CG, Kamenshchik et al. 2001) and generalized Chaplygin gas (GCG, Bento et al. 2002) models have been proposed as possible explanations of the acceleration of the current universe, with the equation of state as follows

$p_{\mathrm{GCG}}=-\frac{A}{\rho_{\mathrm{GCG}}^{\alpha}}$,

where $A$ and $\alpha$ are two parameters to be determined. For the case $\alpha=1$, it corresponds to the original Chaplygin gas (Kamenshchik et al. 2001); if $\alpha=0$, it acts as the cosmological constant $(\Lambda)$. Considering the relativistic energy conservation equation in the framework of Friedmann-Robertson-Walker (FRW) metric, we can obtain

$\rho_{\mathrm{GCG}}=\rho_{\mathrm{GCG}, 0}\left[A_{\mathrm{s}}+\left(1-A_{\mathrm{s}}\right) a^{-3(1+\alpha)}\right]^{\frac{1}{(1+\alpha)}}$,

where $A_{\mathrm{s}} \equiv A / \rho_{\mathrm{GCG}, 0}^{1+\alpha}, \rho_{\mathrm{GCG}, 0}$ is the energy densities of the GCG at present, and the scale factor is related to the redshift by $a=1 /(1+z)$. From Eq. (2), the striking property of the GCG can be found that the energy density behaves as dust like matter at early times; while it behaves like a cosmological constant at late times. Therefore, the GCG model can be regarded as a derivative of the unified dark matter/energy (UDME) scenario (Bento et al. 2004). Until now, the GCG model has been constrained using many different types of observational data, such as Type Ia supernovae (SNe Ia) (Fabris et al. 2002; Makler et al. 2003a; Colistete et al. 2003; Silva \& Bertolami 2003; Cunha et al. 2004; Bertolami et al. 2004; Bento et al. 2006; Wu \& Yu 2007a), cosmic microwave background (CMB) anisotropy (Bento et al. 2003a,b; Bean \& Dore 2003; Amendola et al. 2003), the angular size of the compact radio sources (Zhu 2004), the X-ray gas mass fraction of clusters (Cunha et al. 2004; Makler et al. 2003b), the Hubble parameter versus redshift data (Wu \& Yu 2007b), largescale structure (Bilić et al. 2002; Multamäki et al. 2004), gravitational lensing surveys (Dev et al. 2003, 2004; Chen 2003a,b), age measurements of high- $z$ objects (Alcaniz et al. 2003) and lookback time of galaxy clusters ( $\mathrm{Li}$ et al. 2009); as well as various combinations of data (Wu \& Yu 2007c; Davis et al. 2007; Li et al. 2010; Xu \& Lu 2010).

Gamma-ray bursts (GRBs) have been proposed as distance indicators and regarded as a complementary cosmological probe of the universe at high redshift (Schaefer 2003; Dai et al. 2004; Ghirlanda et al. 2004; Firmani et al. 2005, 2006; Liang \& Zhang 2005; Ghirlanda et al. 2006; Schaefer 2007; Wang et al. 2007; Wright 2007; Amati 2008; Basilakos \& Perivolaropoulos 2008; Mosquera Cuesta et al. 2008a,b; Daly et al. 2008). Owing to the lack of a low-redshift sample, the empirical luminosity relations of GRBs had usually been calibrated by assuming a certain cosmological model with particular model parameters. Liang et al. (2008) presented a completely cosmology-independent method to calibrate GRB luminosity relations with the luminosity distances of GRBs at low redshift interpolated directly from SNe Ia or by other similar approaches (Liang \& Zhang 2008; Kodama et al. 2008; Cardone et al. 2009; Gao et al. 2010; Capozziello \& Izzo 2010). Following the cosmology-independent calibration method, the derived GRB data at high redshift can be used to constrain cosmological models by using the standard Hubble diagram method (Capozziello \& Izzo 2008; Izzo et al. 2009; Wei \& Zhang 2009; Wei 2009; Qi et al. 2009; Wang et al. 2009a,b; Liang et al. 2010a; Wang \& Liang 2010; Liang et al. 2010a; Wei 2010a,b; Liang \& Zhu 2010a; Demianski et al. 2010). Bertolami \& Silva (2006) first studied the GCG model by considering the 
use of GRBs at $1.5<z<5$ calibrated with the bursts at $z<1.5$ as distance markers. The joint analysis with the GCG model of the cosmology-independent GRB data set obtained in Liang et al. (2008) can be found in Wang et al. (2009a) and Freitasa et al. (2010).

Liang et al. (2010a) calibrate GRBs data at high redshift directly from the Union2 compilation of 557 SNe Ia data set (Amanullah et al. 2010) and constrain the Cardassian models by combining the updated GRB data with the joint observations. In this paper, we use the Markov Chain Monte Carlo (MCMC) technique to constrain the GCG model from the latest observational data including the updated distance moduli of the GRBs at high redshift obtained directly from the Union2 set. We combine the GRB data with both the joint observations, such as the Union2 set, the CMB observation from the Wilkinson Microwave Anisotropy Probe (WMAP7; Komatsu et al. 2010) result, and the baryonic acoustic oscillation (BAO) observation from the spectroscopic Sloan Digital Sky Survey (SDSS) data release 7 (DR7) galaxy sample (Percival et al. 2010).

\section{Observational data analysis}

The Union2 compilation consists of data for $557 \mathrm{SNe}$ Ia (Amanullah et al. 2010), and we use the 69 GRBs data compiled by Schaefer (2007). Following Liang et al. (2010a), we use the updated distance moduli of the 42 GRBs at $z>1.4$, which calibrated with the sample at $z \leq 1.4$ by using the linear interpolation method from the Union2 set. For more details for the calculations for GRBs, we refer to Liang et al. (2008) and Liang et al. (2010a). Constraints from SNe Ia and GRB data can be obtained by fitting the distance moduli $\mu(z)$. A distance modulus can be calculated as

$\mu=5 \log \frac{d_{\mathrm{L}}}{\mathrm{Mpc}}+25=5 \log _{10} D_{\mathrm{L}}-\mu_{0}$,

where $\mu_{0}=5 \log _{10}\left[H_{0} /\left(100 \mathrm{~km} \mathrm{~s}^{-1} / \mathrm{Mpc}\right)\right]+42.38$, and the luminosity distance $D_{\mathrm{L}}$ can be calculated using

$D_{\mathrm{L}} \equiv H_{0} d_{\mathrm{L}}=(1+z) \Omega_{\mathrm{k}}^{-1 / 2} \operatorname{sinn}\left[\Omega_{\mathrm{k}}^{1 / 2} \int_{0}^{z} \frac{\mathrm{d} z^{\prime}}{E\left(z^{\prime}\right)}\right]$,

where $\operatorname{sinn}(x)$ is $\sinh$ for $\Omega_{\mathrm{k}}>0$, sin for $\Omega_{\mathrm{k}}<0$, and $x$ for $\Omega_{\mathrm{k}}=0$, and $E(z)=H / H_{0}$, which is determined by the choice of the specific cosmological model. The $\chi^{2}$ value of the observed distance moduli can be calculated by

$\chi_{\mu}^{2}=\sum_{i=1}^{N} \frac{\left[\mu_{\mathrm{obs}}\left(z_{i}\right)-\mu\left(z_{i}\right)\right]^{2}}{\sigma_{\mu, i}^{2}}$,

where $\mu_{\mathrm{obs}}\left(z_{i}\right)$ are the observed distance modulus for the $\mathrm{SNe}$ Ia and/or GRBs at redshift $z_{i}$ with its error $\sigma_{\mu_{\mathrm{i}}} ; \mu\left(z_{i}\right)$ are the theoretical value of the distance modulus from cosmological models. Following the effective approach (Nesseris $\&$ Perivolaropoulos 2005), we marginalize the nuisance parameter $\mu_{0}$ by minimizing $\hat{\chi}_{\mu}^{2}=C-B^{2} / A$, where $A=$ $\sum 1 / \sigma_{\mu_{i}}^{2}, \quad B=\sum\left[\mu_{\mathrm{obs}}\left(z_{i}\right)-5 \log _{10} D_{\mathrm{L}}\right] / \sigma_{\mu_{i}}^{2}$, and $C=$ $\sum\left[\mu_{\mathrm{obs}}\left(z_{i}\right)-5 \log _{10} D_{\mathrm{L}}\right]^{2} / \sigma_{\mu_{i}}^{2}$.

For the CMB observation, we use the data set including the acoustic scale $\left(l_{\mathrm{a}}\right)$, the shift parameter $(R)$, and the redshift of recombination $\left(z_{*}\right)$, which provide an efficient summary of CMB data as far as cosmological constraints go. The acoustic scale can be expressed as

$l_{\mathrm{a}}=\pi \frac{\Omega_{\mathrm{k}}^{-1 / 2} \operatorname{sinn}\left[\Omega_{\mathrm{k}}^{1 / 2} \int_{0}^{z_{*}} \frac{\mathrm{d} z}{E(z)}\right] / H_{0}}{r_{\mathrm{s}}\left(z_{*}\right)}$, where $r_{\mathrm{s}}\left(z_{*}\right)=H_{0}^{-1} \int_{z_{*}}^{\infty} c_{\mathrm{s}}(z) / E(z) \mathrm{d} z$ is the comoving sound horizon at photo-decoupling epoch. The shift parameters can be expressed as

$R=\Omega_{\mathrm{M} 0}^{1 / 2} \Omega_{\mathrm{k}}^{-1 / 2} \operatorname{sinn}\left[\Omega_{\mathrm{k}}^{1 / 2} \int_{0}^{z_{*}} \frac{\mathrm{d} z}{E(z)}\right]$.

The redshift of recombination can be given by (Hu \& Sugiyama 1996)

$z_{*}=1048\left[1+0.00124\left(\Omega_{\mathrm{b}} h^{2}\right)^{-0.738}\left(1+g_{1}\left(\Omega_{\mathrm{M} 0} h^{2}\right)^{g_{2}}\right)\right]$,

where $g_{1}=0.0783\left(\Omega_{\mathrm{b}} h^{2}\right)^{-0.238}\left(1+39.5\left(\Omega_{\mathrm{b}} h^{2}\right)^{-0.763}\right)^{-1}$ and $g_{2}=$ $0.560\left(1+21.1\left(\Omega_{\mathrm{b}} h^{2}\right)^{1.81}\right)^{-1}$. From the WMAP7 measurement, the best-fit values of the data set $\left(l_{\mathrm{a}}, R, z_{*}\right)$ are (Komatsu et al. 2010)

$\overline{\mathbf{P}}_{\mathrm{CMB}}=\left(\begin{array}{c}\bar{l}_{\mathrm{a}} \\ \bar{R} \\ \bar{z}_{*}\end{array}\right)=\left(\begin{array}{c}302.09 \pm 0.76 \\ 1.725 \pm 0.018 \\ 1091.3 \pm 0.91\end{array}\right)$.

The $\chi^{2}$ value of the CMB observation can be expressed as (Komatsu et al. 2010)

$\chi_{\mathrm{CMB}}^{2}=\Delta \mathbf{P}_{\mathrm{CMB}}^{\mathrm{T}} \mathbf{C}_{\mathrm{CMB}}{ }^{-1} \Delta \mathbf{P}_{\mathrm{CMB}}$,

where $\Delta \mathbf{P}_{\mathrm{CMB}}=\mathbf{P}_{\mathrm{CMB}}-\overline{\mathbf{P}}_{\mathrm{CMB}}$, and the corresponding inverse covariance matrix is

$\mathbf{C}_{\mathrm{CMB}}{ }^{-1}=\left(\begin{array}{ccc}2.305 & 29.698 & -1.333 \\ 29.698 & 6825.270 & -113.180 \\ -1.333 & -113.180 & 3.414\end{array}\right)$

For the BAO observation, we use the measurement of the BAO distance ratio $\left(d_{z}\right)$ at $z=0.2$ and $z=0.35$ (Percival et al. 2010), which can be expressed as

$d_{z}=\frac{r_{\mathrm{s}}\left(z_{\mathrm{d}}\right)}{D_{V}\left(z_{\mathrm{BAO}}\right)}$

where the distance scale $D_{V}$ is given by (Eisenstein et al. 2005)

$D_{V}\left(z_{\mathrm{BAO}}\right)=\frac{1}{H_{0}}\left[\frac{z_{\mathrm{BAO}}}{E\left(z_{\mathrm{BAO}}\right)}\left(\int_{0}^{z_{\mathrm{BAO}}} \frac{\mathrm{d} z}{E(z)}\right)^{2}\right]^{1 / 3}$,

and $r_{\mathrm{s}}\left(z_{\mathrm{d}}\right)$ is the comoving sound horizon at the drag epoch at which baryons were released from photons, $z_{\mathrm{d}}$ can be given by (Eisenstein \& Hu 1998)

$z_{\mathrm{d}}=\frac{1291\left(\Omega_{\mathrm{M} 0} h^{2}\right)^{0.251}}{\left[1+0.659\left(\Omega_{\mathrm{M} 0} h^{2}\right)^{0.828}\right]}\left[\left(1+b_{1}\left(\Omega_{\mathrm{b}} h^{2}\right)^{b_{2}}\right)\right]$,

where $b_{1}=0.313\left(\Omega_{\mathrm{M} 0} h^{2}\right)^{-0.419}\left[1+0.607\left(\Omega_{\mathrm{M} 0} h^{2}\right)^{0.674}\right]^{-1}$ and $b_{2}=0.238\left(\Omega_{\mathrm{M} 0} h^{2}\right)^{0.223}$. From SDSS data release 7 (DR7) galaxy sample, the best-fit values of the data set $\left(d_{0.2}, d_{0.35}\right)$ are (Percival et al. 2010)

$\overline{\mathbf{P}}_{\mathrm{BAO}}=\left(\begin{array}{l}\bar{d}_{0.2} \\ \bar{d}_{0.35}\end{array}\right)=\left(\begin{array}{l}0.1905 \pm 0.0061 \\ 0.1097 \pm 0.0036\end{array}\right)$.

The $\chi^{2}$ value of the BAO observation from SDSS DR7 can be expressed as (Percival et al. 2010)

$\chi_{\mathrm{BAO}}^{2}=\Delta \boldsymbol{P}_{\mathrm{BAO}}^{\mathrm{T}} \boldsymbol{C}_{\mathrm{BAO}}{ }^{-1} \Delta \boldsymbol{P}_{\mathrm{BAO}}$,

where the corresponding inverse covariance matrix is

$\boldsymbol{C}_{\mathrm{BAO}}{ }^{-1}=\left(\begin{array}{cc}30124 & -17227 \\ -17227 & 86977\end{array}\right)$. 


\section{Constraints on the GCG Model via MCMC method}

We consider a flat universe filled with the GCG component and the baryon matter component. From the Friedmann equation $H^{2}=(8 \pi G / 3)\left(\rho_{\mathrm{b}}+\rho_{\mathrm{GCG}}\right)$, we find that

$$
\begin{aligned}
E^{2}\left(z ; A_{\mathrm{s}}, \alpha\right) \equiv \frac{H^{2}}{H_{0}^{2}}= & \Omega_{\mathrm{b}}(1+z)^{3}+\left(1-\Omega_{\mathrm{b}}\right) \\
& \times\left[A_{\mathrm{s}}+\left(1-A_{\mathrm{s}}\right)(1+z)^{3(1+\alpha)}\right]^{\frac{1}{(1+\alpha)}},
\end{aligned}
$$

where $\Omega_{\mathrm{b}}$ represents the fractional contribution of baryon matter. The effective matter density in the GCG model can be given by (Bento et al. 2004; Wu \& Yu 2007c)

$\Omega_{\mathrm{m}}=\Omega_{\mathrm{b}}+\left(1-\Omega_{\mathrm{b}}\right)\left(1-A_{\mathrm{s}}\right)^{\frac{1}{(1+\alpha)}}$.

To combine GRB data with the SNe Ia data and constrain cosmological models, we follow the simple method of avoiding any correlation between the SNe Ia data and the GRB data: the $40 \mathrm{SNe}$ points used in the interpolating procedure are excluded from the Union2 SNe Ia sample used to derive the joint constraints (Liang et al. 2010a,b). The 42 GRBs and the reduced $517 \mathrm{SNe}$ Ia, CMB, BAO are all effectively independent, therefore we can combine the results by simply multiplying the likelihood functions. The total $\chi^{2}$ with the $\mathrm{SNe}+\mathrm{GRBs}+\mathrm{CMB}+$ $\mathrm{BAO}$ dataset is

$\chi^{2}=\hat{\chi}_{\{\mathrm{SNe}, \mathrm{GRBs}\}}^{2}+\chi_{\mathrm{CMB}}^{2}+\chi_{\mathrm{BAO}}^{2}$.

We perform a global fitting to determine the cosmological parameters using the Markov Chain Monte Carlo (MCMC) method. In adopting the MCMC approach, we generate using Monte Carlo methods a chain of sample points distributed in the parameter space according to the posterior probability, using the Metropolis-Hastings algorithm with uniform prior probability distribution. In the parameter space formed by the constraint cosmological parameters, a random set of initial values of the model parameters is chosen to calculate the $\chi^{2}$ or the likelihood. Whether the set of parameters can be accepted as an effective Markov chain or not is determined by the MetropolisHastings algorithm. The accepted set not only forms a Markov chain, but also provides a starting point for the next process. We then repeat this process until the established convergence accuracy can be satisfied. The convergence is tested by checking the so-called worst e-values [the variance(mean)/mean(variance) of $1 / 2$ chains $] R-1<0.005$.

Our MCMC code is based on the publicly available CosmoMC package (Lewis \& Bridle 2002), and we generated eight chains after setting $R-1=0.001$ to guarantee the accuracy of this work. We show the 1-D probability distribution of each parameter in the MCMC method $\left(\Omega_{\mathrm{b}} h^{2}, A_{\mathrm{S}}, \alpha, \Omega_{\Lambda}\right.$, Age/Gyr, $\Omega_{\mathrm{m}}, H_{0}$ ) and 2-D plots for parameters between each other for the GCG model with $\mathrm{SNe}+\mathrm{GRBs}+\mathrm{CMB}+\mathrm{BAO}$ in Fig. 1 (Age/Gyr is the cosmic age, in units of Gyr). The best-fit values of the GCG model parameters with the joint observational data are $A_{\mathrm{S}}=0.7475_{-0.0539}^{+0.0556}(1 \sigma)_{-0.0816}^{+0.0794}(2 \sigma), \alpha=$ $-0.0256_{-0.1326}^{+0.176}(1 \sigma)_{-0.1907}^{+0.2730}(2 \sigma)$, and the effective matter density $\Omega_{\mathrm{m}}=0.2629_{-0.0153}^{+0.0155}(1 \sigma)_{-0.0223}^{+0.0236}(2 \sigma)$. For comparison, fitting results from the joint data of $557 \mathrm{SNe}$ Ia, the CMB and BAO without GRBs and 42 GRBs, the CMB and BAO without SNe Ia are given in Figs. 2 and 3. We present the best-fit values of each parameter with the $1-\sigma$ and $2-\sigma$ uncertainties, as well as $\chi_{\min }^{2}$, in Table 1.

From Figs. 1-3 and Table 1, it is shown that the cosmological constant $(\alpha=0)$ is allowed at the $1-\sigma$ confidence level, and the

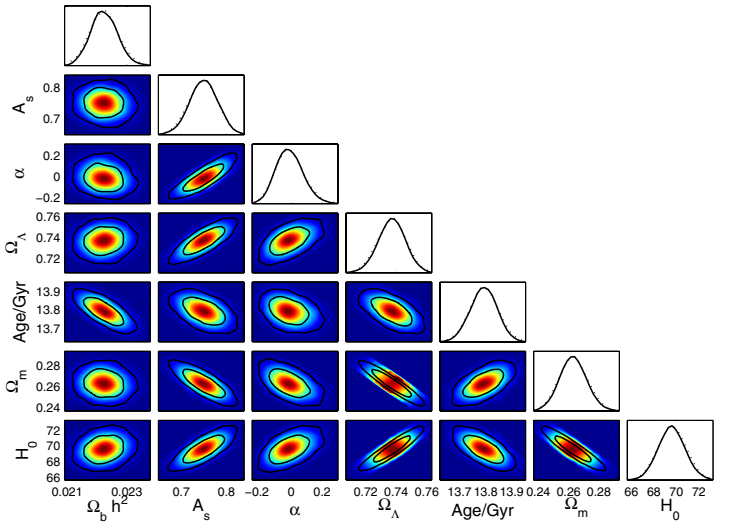

Fig. 1. The 2-D regions and 1-D marginalized distribution with the 1- $\sigma$ and $2-\sigma$ contours of parameters $\Omega_{\mathrm{b}} h^{2}, A_{\mathrm{S}}, \alpha, \Omega_{\Lambda}$, Age/Gyr, $\Omega_{\mathrm{m}}$, and $H_{0}$ in GCG model, for the data sets $\mathrm{SNe}+\mathrm{GRBs}+\mathrm{CMB}+\mathrm{BAO}$.

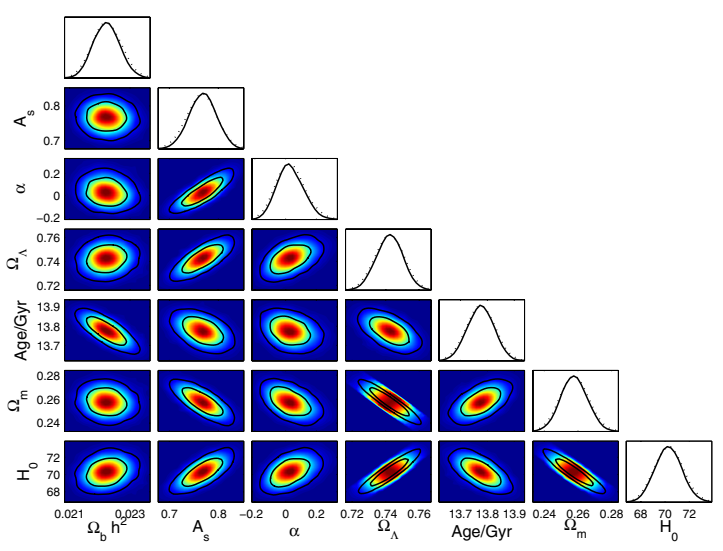

Fig. 2. The 2-D regions and 1-D marginalized distribution with the 1- $\sigma$ and $2-\sigma$ contours of parameters $\Omega_{\mathrm{b}} h^{2}, A_{\mathrm{S}}, \alpha, \Omega_{\Lambda}$, Age/Gyr, $\Omega_{\mathrm{m}}$, and $H_{0}$ in GCG model, for the data sets $\mathrm{SNe}+\mathrm{CMB}+\mathrm{BAO}$.

original Chaplygin gas model $(\alpha=1)$ is ruled out at $95.4 \%$ confidence level, which are both consistent with that obtained in $\mathrm{Wu}$ \& Yu (2007c), and Li et al. (2009). We can find that GRBs can provide strong constraints when combined with $\mathrm{CMB}$ and $\mathrm{BAO}$ data without SNe Ia, which has also been noted by Liang, Wu \& Zhu (2010), Liang \& Zhu (2010a), and Gao et al. (2010). In additoin, the constraining results in this work with the joint observational data including GRBs are more stringent than previous results for constraining GCG model parameters with GRBs and/or other combined observations (e.g. Wang et al. 2009a,b; Freitasa et al. 2010; Davis et al. 2007; Wu \& Yu 2007c; Li et al. 2009; Li et al. 2010; Xu \& Lu 2010).

\section{Conclusions}

By using the Markov Chain Monte Carlo method, we have constrained on the generalized Chaplygin gas (GCG) model with the cosmology-independent GRBs, as well as the Union2 SNe Ia set, the CMB observation from WMAP7 result, and the BAO observation from SDSS DR7 galaxy sample. With the joint observational data, the best-fit values of the GCG model parameters are $A_{\mathrm{S}}=0.7475_{-0.0539}^{+0.0556}(1 \sigma)_{-0.0816}^{+0.0794}(2 \sigma), \alpha=$ $-0.0256_{-0.1326}^{+0.1760}(1 \sigma)_{-0.1907}^{+0.2730}(2 \sigma)$, and the effective matter density $\Omega_{\mathrm{m}}=0.2629_{-0.0153}^{+0.0155}(1 \sigma)_{-0.0223}^{+0.0236}(2 \sigma)$, which are more stringent than previous results for constraining the GCG model parameters obtained using data of GRBs and/or other combinations of observations. 
Table 1. The best-fit values of parameters $\Omega_{\mathrm{b}} h^{2}, A_{\mathrm{S}}, \alpha, \Omega_{\Lambda}$, Age/Gyr, $\Omega_{\mathrm{m}}$, and $H_{0}$ for the GCG model with the 1- $\sigma$ and 2- $\sigma$ uncertainties, as well as $\chi_{\min }^{2}$, for the data sets $\mathrm{SNe}+\mathrm{CMB}+\mathrm{BAO}, \mathrm{SNe}+\mathrm{GRBs}+\mathrm{CMB}+\mathrm{BAO}$, and $\mathrm{GRBs}+\mathrm{CMB}+\mathrm{BAO}$, respectively.

\begin{tabular}{|c|c|c|c|}
\hline & \multicolumn{3}{|c|}{ The GCG Model } \\
\hline & $\mathrm{SNe}+\mathrm{GRBs}+\mathrm{CMB}+\mathrm{BAO}$ & $\mathrm{SNe}+\mathrm{CMB}+\mathrm{BAO}$ & GRBs+CMB+BAO \\
\hline$\Omega_{\mathrm{b}} h^{2}$ & $0.0222_{-0.0007}^{+0.0008}(1 \sigma)_{-0.0012}^{+0.0009}(2 \sigma)$ & $0.0222_{-0.0007}^{+0.0008}(1 \sigma)_{-0.0009}^{+0.0012}(2 \sigma)$ & $0.0222_{-0.0007}^{+0.0008}(1 \sigma)_{-0.0009}^{+0.0012}(2 \sigma)$ \\
\hline$A_{\mathrm{S}}$ & $0.7475_{-0.0539}^{+0.0556}(1 \sigma)_{-0.0816}^{+0.0744}(2 \sigma)$ & $0.7668_{-0.0510}^{+0.0492}(1 \sigma)_{-0.0751}^{+0.0711}(2 \sigma)$ & $0.7665_{-0.1427}^{+0.1358}(1 \sigma)_{-0.2069}^{+0.1835}(2 \sigma)$ \\
\hline$\alpha$ & $-0.0256_{-0.1326}^{+0.1760}(1 \sigma)_{-0.1907}^{+0.2730}(2 \sigma)$ & $0.0198_{-0.1348}^{+0.1694}(1 \sigma)_{-0.1960}^{+0.2606}(2 \sigma)$ & $0.0184_{-0.2922}^{+0.5293}(1 \sigma)_{-0.3929}^{+0.9024}(2 \sigma)$ \\
\hline$\Omega_{\Lambda}$ & $0.7371_{-0.0155}^{+0.0153}(1 \sigma)_{-0.0236}^{+0.0223}(2 \sigma)$ & $0.7425_{-0.0146}^{+0.0137}(1 \sigma)_{-0.0216}^{+0.0200}(2 \sigma)$ & $0.7425_{-0.0415}^{+0.0360}(1 \sigma)_{-0.0617}^{+0.0497}(2 \sigma)$ \\
\hline Age/Gyr & $13.79_{-0.09}^{+0.09}(1 \sigma)_{-0.14}^{+0.13}(2 \sigma)$ & $13.77_{-0.09}^{+0.09}(1 \sigma)_{-0.13}^{+0.13}(2 \sigma)$ & $13.77_{-0.13}^{+0.17}(1 \sigma)_{-0.20}^{+0.26}(2 \sigma)$ \\
\hline$\Omega_{\mathrm{m}}$ & $0.2629_{-0.0153}^{+0.0155}(1 \sigma)_{-0.0223}^{+0.0236}(2 \sigma)$ & $0.2575_{-0.0146}^{+0.0137}(1 \sigma)_{-0.0200}^{+0.0216}(2 \sigma)$ & $0.2575_{-0.0360}^{+0.0415}(1 \sigma)_{-0.0497}^{+0.0617}(2 \sigma)$ \\
\hline$H_{0}$ & $69.56_{-2.01}^{+2.14}(1 \sigma)_{-2.91}^{+3.19}(2 \sigma)$ & $70.29_{-1.98}^{+1.95}(1 \sigma)_{-2.84}^{+2.91}(2 \sigma)$ & $70.29_{-5.08}^{+5.47}(1 \sigma)_{-7.15}^{+7.87}(2 \sigma)$ \\
\hline$\chi_{\min }^{2}$ & 502.266 & 544.828 & 36.930 \\
\hline
\end{tabular}

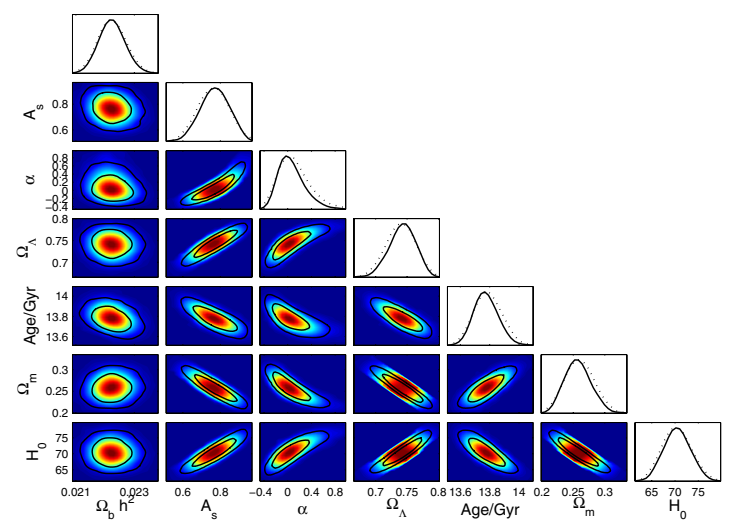

Fig. 3. The 2-D regions and 1-D marginalized distribution with the $1-\sigma$ and 2- $\sigma$ contours of parameters $\Omega_{\mathrm{b}} h^{2}, A_{\mathrm{S}}, \alpha, \Omega_{\Lambda}$, Age/Gyr, $\Omega_{\mathrm{m}}$, and $H_{0}$ in GCG model, for the data sets GRBs+CMB+BAO.

Acknowledgements. We thank Yun Chen, Shuo Cao, Hao Wang, Yan Dai, Chunhua Mao, Fang Huang, Yu Pan, Jing Ming, Kai Liao and Dr. Yi Zhang for discussions. This work was supported by the National Science Foundation of China under the Distinguished Young Scholar Grant 10825313, the Key Project Grants 10533010, and by the Ministry of Science and Technology national basic science Program (Project 973) under grant No. 2007CB815401. L.X. acknowledges partial supports by NSF (10703001), SRFDP (20070141034) of P.R. China and the Fundamental Research Funds for the Central Universities (DUT10LK31).

\section{References}

Amanullah, R., Lidman, C., Rubin, D., et al. 2010, ApJ, 716, 712

Amati, L., Guidorzi, C., Frontera, F., et al. 2008, MNRAS, 391, 577

Alcaniz, J. S., Jain, D., \& Dev, A. 2003, Phys. Rev. D, 67, 043514

Amendola, L., Finelli, L. F., Burigana, C., \& Carturan, D. 2003, JCAP, 0307, 005

Basilakos, S., \& Perivolaropoulos, L. 2008, MNRAS, 391, 411

Bean, R., \& Dore, O. 2003, Phys. Rev. D, 68, 023515

Bento, M. C., Bertolami, O., \& Sen, A. A. 2002, Phys. Rev. D, 66, 043507

Bento, M. C., Bertolami, O., \& Sen, A. A. 2003a, Phys. Rev. D, 67, 063003

Bento, M. C., Bertolami, O., \& Sen, A. A. 2003b, Phys. Lett. B, 575, 172

Bento, M. C., Bertolami, O., \& Sen, A. A. 2004, Phys. Rev. D, 70, 083519

Bento, M. C., Bertolami, O., Reboucas, M. J., \& Silva, P. T. 2006, Phys. Rev. D, 73, 043504

Bertolami, O., \& Silva, P. T. 2006, MNRAS, 365, 1149

Bertolami, O., Sen, A. A., Sen, S., \& Silva, P. T. 2004, MNRAS, 353, 329

Bilić, N., Tupper, G. B., \& Viollier, R. D. 2002, Phys. Lett. B, 535, 17

Capozziello, S., \& Izzo, L. 2008, A\&A, 490, 31

Capozziello, S., \& Izzo, L. 2010 [arXiv: 1003. 5319]

Cardone, V. F., Capozziello, S., \& Dainotti, M. G. 2009, MNRAS, 400, 775

Chen, D. M. 2003a, ApJ, 587, L55

Chen, D. M. 2003b, A\&A, 397, 415

Colistete Jr, R., Fabris, J. C., Gonalves, S. V. B., \& de Souza, P. E. 2003, Int. J. Mod. Phys. D, 13, 669

Cunha, J. V., Lima, J. A. S., \& Alcaniz, J. S. 2004, Phys. Rev. D, 69, 083501

Dai, Z. G., Liang, E. W., \& Xu, D. 2004, ApJ, 612, L101
Davis, T. M., Mörtsell, E., Sollerman, J., et al. 2007, ApJ, 666, 716D Daly, R. A., Djorgovski, S. G., Freeman, K. A., et al. 2008, ApJ, 677, 1 Demianski, M., Piedipalumbo, E., \& Rubano, C. 2010 [arXiv: 1010.0855] Dev, A., Jain, D., \& Alcaniz, J. S. 2003, Phys. Rev. D, 67, 023515 Dev, A., Jain, D., \& Alcaniz, J. S. 2004, A\&A, 417, 847 Eisenstein, D., \& Hu, W. 1998, ApJ, 496, 605 Eisenstein, D. J., Zehavi, I., Hogg, D. W., et al. 2005, ApJ, 633, 560 Fabris, J. C., Goncalves, S. V. B., \& de Souza, P. E. 2002, unpublished [arXiv: astro-ph/0207430]

Firmani, C., Ghisellini, G., Ghirlanda, G., \& Avila-Reese, V. 2005, MNRAS, 360, L1

Firmani, C., Avila-Reese, V., Ghisellini, G., \& Ghirlanda, G. 2006, MNRAS, 372, L28

Freitasa, R. C., Gonçalves, S. V. B., \& Velten, H. E. S., et al. 2010 [arXiv: 1004.5585$]$

Gao, H., Liang, N., \& Zhu, Z. H. 2010 [arXiv: 1003. 5755]

Ghirlanda, G., Ghisellini, G., \& Lazzati, D. 2004, ApJ, 613, L13

Ghirlanda, G., Ghisellini, G., \& Firmani, C. 2006, New J. Phys, 8, 123

Hu, W., \& Sugiyama, N. 1996, ApJ, 471, 542

Izzo, L., Capozziello, S., Covone, G., \& Capaccioli, M. 2009, A\&A, 508, 63 Kamenshchik, A., Moschella, U., \& Pasquier, V. 2001, Phys. Lett. B, 511, 265

Kodama, Y., Yonetoku, D., Murakami, T., et al. 2008, MNRAS, 391, L1

Komatsu, E., Smith, K. M., Dunkley, J., et al. [WMAP Collaboration] 2010 [arXiv: 1001.4538]

Lewis, A., \& Bridle, S. 2002, Phys. Rev. D, 66,103511 http: //cosmologist. info/cosmomc/

Li, M., Li, X.-D., \& Zhang, X. 2010, ScChG, 53, 1631

Li, Z., Wu, P., \& Yu, H. 2009, JCAP, 09, 017

Liang, E. W., \& Zhang, B. 2005, ApJ, 633, 603

Liang, N., \& Zhang, S. N. 2008, AIP Conf. Proc., 1065, 367

Liang, N., \& Zhu, Z.-H. 2010 [arXiv: 1010.2681]

Liang, N., Xiao, W. K., Liu, Y., \& Zhang, S. N. 2008, ApJ, 685, 354

Liang, N., Wu, P., \& Zhang, S. N. 2010a, Phys. Rev. D, 81, 083518

Liang, N., Wu, P., \& Zhu, Z.-H. 2010b [arXiv: 1006. 1105]

Makler, M., Oliveira, S. Q., \& Waga, I. 2003a, Phys. Lett. B, 555,

Makler, M., Oliveira, S. Q., \& Waga, I. 2003b, Phys. Rev. D, 68, 123521

Mosquera Cuesta, H. J., Dumet, M. H., \& Furlanetto, C. 2008a, JCAP, 07, 04

Mosquera Cuesta, H. J., Turcati, R., Furlanetto, C., et al. 2008b, A\&A, 487, 47

Multamäki, T., Manera, M., \& Gaztañaga, E. 2004, Phys. Rev. D, 69, 023004

Nesseris, S., \& Perivolaropoulos, L. 2005, Phys. Rev. D, 72, 123519

Percival, W. J., Reid, B. A., Eisenstein, D. J., et al. 2010, MNRAS, 401, 2148

Qi, S., Lu, T., \& Wang, F. Y. 2009, MNRAS, 398, L78

Schaefer, B. E. 2003, ApJ, 583, L67

Schaefer, B. E. 2007, ApJ, 660, 16

Silva, P. T., \& Bertolami, O. 2003, ApJ, 599, 829

Wang, F. Y., Dai, Z. G., \& Zhu, Z.-H. 2007, ApJ, 667, 1

Wang, F. Y., Dai, Z. G., \& Qi, S. 2009a, A\&A, 507, 53

Wang, F. Y., Dai, Z. G., \& Qi, S. 2009b, RAA, 9, 547

Wang, T. S., \& Liang, N. 2010, ScChG, 53, 1720

Wei, H. 2009, EPJC, 60, 449

Wei, H. 2010a, JCAP, 08, 020

Wei, H. 2010b, Phys. Lett. B, 692, 167

Wei, H., \& Zhang, S. N. 2009, EPJC, 63, 139

Wright, E. L. 2007, ApJ, 664, 633

Wu, P., \& Yu, H. 2007a, ApJ, 658, 663

Wu, P., \& Yu, H. 2007b, Phys. Lett. B, 644, 16

Wu, P., \& Yu, H. 2007c, JCAP, 03, 015

Xu, L., \& Lu, J. 2010, JCAP, 03, 025

Zhu, Z.-H. 2004, A\&A, 423, 421 